EPJ Web of Conferences 103, 08002 (2015)

DOI: $10.1051 /$ epjconf/ 201510308002

(C) Owned by the authors, published by EDP Sciences, 2015

\title{
Nonresonant Multiple-Pulse Control of Molecular Motions in Liquid
}

\author{
V.G. Nikiforov ${ }^{\mathrm{a}}$ \\ Zavoisky Kazan Physical-Technical Institute, 10/7Sibirskiy Tract Str.. Kazan 420029, Russia
}

\begin{abstract}
We propose the implementation of the multiple-pulse excitation for manipulation of the molecular contributions to the optically-heterodyne-detected optical-Kerr-effect. The key parameters controlling the specificity of the multiple-pulse excitation scenarios are the pulses durations, the delays between pulses, the relation between the pump pulses amplitudes and the pulses polarizations. We model the high-order optical responses and consider some principles of the scenarios construction. We show that it is possible to adjust the excitation scenario in such a way that the some responses can be removed from detected signal along with the enhancement of the interested response amplitude. The theoretical analysis and first experimental data reveal that the multiple-pulse excitation technique can be useful for the selective spectroscopy of the molecular vibrations and rotations in liquid.
\end{abstract}

Key words: multiple-pulse spectroscopy, coherent control, ultrafast optical Kerr effect.

The two reasons make the ultrafast optically-heterodyne-detected optical Kerr effect (OHD-OKE) a popular tool for monitoring the ultrafast dynamics of liquids. First, the implementation of the experiment is relatively straightforward. Second, the experiment provides data of extremely high signal-to-noise ratio. As a result, the OHD-OKE method is applied in a wide range of problems in condensed phase dynamics. It is clear that other methods (for example, Raman scattering) yield the same spectra, but the OHD-OKE spectroscopy reveals a rather complete picture of the subpicosecond and picosecond orientational molecular motions, along with a less well characterized contribution to the relaxation from the intermolecular interactions.

The OHD-OKE optical experiments measure the nonlinear response function of the sample [1, 2], which comprises two components: an effectively instantaneous electronic contribution and a nuclear response functions. The nuclear terms arise from the coherent intramolecular vibrations, the molecular reorientations, the intermolecular translational, rotational collisions and the molecular librations [3, 4].

We propose the using of high-order nonlinear optical technique permitting the manipulation of the different molecular motions [5,6]. It is known that the femtosecond laser technique is one of promising tools for the control of the molecular dynamics. Primarily it is due to the excitation time comparable to the period of the intermolecular oscillations. We use a multiple-pulse excitation - the one of the actual methods for the control of the molecular dynamics. For example, a multiple-pulse excitation made it possible to control the coherent phonon oscillations in a number of solid materials [7-10]. In a liquid, molecular responses at room temperature have subpicosecond relaxation times.

\footnotetext{
${ }^{\mathrm{a}}$ Corresponding author: vgnik@mail.ru
} 
This complicates the multiple-pulse excitation by the requirement of using the femtosecond time resolution technique.

In our case the excitation scenarios are specified by the parameters of the sequence of the pump pulses: the pulses intensities, the delays between the pulses etc. This permits the realization of various modes for the control of the molecular dynamics and, in such a way, the manipulation of the molecular responses contributions to the entire OHD-OKE signal. For the first time we implemented the double-pulse excitation to control the amplitude of the vibrational response in chloroform and carbon tetrachloride $[11,12]$. We analyze also our results of the double-pulse control of the coherent vibrational and rotational molecular motions in acetonitrile [13] and discuss the aspects of the ultrafast non-resonant multiple-pulse selective spectroscopy of the molecular motions in liquid.

\section{References}

1. Mukamel, Principles of Nonlinear Optical Spectroscopy (Oxford University Press, New York, 1995)

2. D.McMorrow, W.T. Lotshaw, G. A. Kenney-Wallace, IEEE J. Quantum Electron., 24, 443 (1988)

3. J.S. Fridman, M.C. Lee, C.Y. She, Chem. Phys. Lett., 186, 161 (1991)

4. T.H. Huang, C.C. Hsu, T.H. Wei, M.J. Chen, W.S. Tse, H.P. Chiang, C.T. Kuo, S. Chang, X.J. Gu, Molecular Physics, 96, 389 (1999)

5. Y. Tanimura, S. Mukamel, J. Chem. Phys., 99, 9496 (1993)

6. T. Steffen, J.T. Fourkas, K. Duppen, J.Chem. Phys., 1057364 (1996)

7. H. Hase, K. Mizoguchi, H. Harima, S. Nakashima, M. Tani, K. Sakai, M. Hangyo, Appl. Phys. Lett., 69, 2474 (1996)

8. M. Hase, M. Kitajima, S. Nakashima, K. Mizoguchi, Appl. Phys. Lett., 83, 4921 (2003)

9. A.Q. Wu, X. Xu, Appl. Phys. Lett., 90, 251111 (2007)

10. C.M. Liebig, Y. Wang, X. Xu, Optics Express, 18, 20498 (2010)

11. V.G. Nikiforov, A.G. Shmelev, G.M. Safiullin, V.S. Lobkov, Quantum Electron., 42, 332 (2012)

12. V.G. Nikiforov, A.G. Shmelev, G.M. Safiullin, V.S. Lobkov, Appl. Phys. Lett., 100, 081904-1 (2012)

13. V.G. Nikiforov, A.G. Shmelev, G.M. Safiullin, V.S. Lobkov, Chem. Phys. Lett., 592, 196 (2014) 